\title{
Immunogenic Comparison of Two Coupling Methods of Marine Polysaccharide to Bovine Serum Albumin
}

\author{
Li Gan, ${ }^{a}$ Xianliang XIN, ${ }^{a}$ Meiyu GenG, ${ }^{*}, a$ and Jian DING ${ }^{b}$ \\ ${ }^{a}$ Department of Pharmacology, Marine Drug and Food Institute, Ocean University of China; Qingdao, 266003, P. R. \\ China; and ${ }^{b}$ Division of Antitumor Pharmacology, State Key Laboratory of Drug Research, Shanghai Institute of Materia \\ Medica, Shanghai Institutes for Biological Sciences, Chinese Academy of Sciences; Shanghai, 201203, P. R. China. \\ Received March 15, 2004; accepted June 1, 2004
}

Two conjugates of marine polysaccharide (MPS) and bovine serum albumin (BSA) were prepared using two methods, periodate oxidation and reductive amination, with the intent of enhancing its immunogenicity. Sera samples from Balb/c mice immunized with the products named MPS-BSAp and MPS-BSAr respectively were evaluated by enzyme-linked-immunosorbent assay (ELISA). The results showed that mice immunized with MPS-BSAp produced antibodies not against MPS but rather against MPS-BSAp, while the mice immunized with MPS-BSAr produced high titer antibodies only specific for MPS. The difference was attributed to the fact that the epitopes of MPS had been changed in the coupling process by periodate oxidation. A mouse immunized with MPS-BSAr was chosen to prepare monoclonal antibodies (mAbs) specific for polysaccharide MPS. A hybridoma cell line that secreted monoclonal antibody recognizing specifically polysaccharide MPS was established.

Key words marine polysaccharide; monoclonal antibody; periodate oxidation; reductive amination

Much of the 25 years since Köhler and Milstein first described the preparation of monoclonal antibodies (mAbs) has been spent trying to develop mAbs for treating human diseases and as research tools in biological and clinical studies. ${ }^{1)}$ To produce specific antibodies in animals, it is crucial to prepare immunogens with high immunogenicity. It has been reported that the immunogenicities of carbohydrates including polysaccharides and oligosaccharides are very weak, but animals will produce specific antibodies for carbohydrates immunized with proteoglycans, or artificial immunogens of carbohydrate-carrier conjugates. ${ }^{2-9)}$ Numerous methods for coupling carbohydrates to carriers have been reported, and protein is generally used as a carrier. ${ }^{10-17)}$

Marine polysaccharide (MPS), an anti-Alzheimer's disease drug candidate, is currently undergoing preclinical evaluation. The preparation of mAbs specific for MPS is both biologically and clinically available. MPS bearing mannuronic acid blocks is an acidic polysaccharide extracted from marine brown algae and has an average molecular weight of about $8000 \mathrm{Da}$. MPS or even brown algae alone could not induce specific antibodies in Balb/c mice. In order to obtain mAbs of MPS, MPS-BSA conjugates were prepared as artificial immunogens by two coupling methods, periodate oxidation and reductive amination, with which we would search for an artificial immunogen with high immunogenicity. Balb/c mice were then immunized with these two conjugates named MPS-BSAp and MPS-BSAr respectively. Antisera from mice were tested by enzymelinked-immunosorbent assay (ELISA) to evaluate immunogenicities of the two conjugates and specificities of the antibodies. In the present study, we attempted to determine which method for conjugating MPS to BSA was more effective for inducing high titer antibodies that could react only with native MPS.

\section{MATERIALS AND METHODS}

Materials MPS was provided by Marine Drug and Food Institute, Ocean University of China (Qingdao, China). Balb/c mice were obtained from Animal Center of Shangdong University, China. Bovine serum albumin (BSA), ovalbumin (OVA), gelatin, horseradish peroxidase (HRP) conjugated goat anti-mouse IgG $(\mathrm{H}+\mathrm{L})$, sodium periodate $\left(\mathrm{NaIO}_{4}\right)$, sodium cyanoborohydride $\left(\mathrm{NaBH}_{3} \mathrm{CN}\right)$, and 3,3',5,5'-tetramethylbenzidine (TMB) were purchased from Sigma (St. Louis, MO, U.S.A.). Flat-bottomed polystyrene microplates were purchased from Costar (NY, U.S.A.). ELISA reader (Rainbow) was purchased from Tecan (Durham, Austria). Immunowash (1575) was purchased from Bio-Rad (Hercules, CA, U.S.A.).

Preparation of MPS-BSAp by Periodate Oxidation There are vicinal hydroxyl groups on the sugar unit of MPS, which can be changed into aldehyde groups by periodate oxidation. The aldehyde groups can be subsequently coupled to amino groups of BSA in the presence of $\mathrm{NaBH}_{3} \mathrm{CN}$. Five hundred micrograms of MPS dissolved in $12 \mathrm{ml}$ of $0.02 \mathrm{M}$ sodium acetate, $\mathrm{pH} 4.0$, was added to $12 \mathrm{ml} 0.1 \mathrm{M}$ fresh $\mathrm{NaIO}_{4}$ solution. After agitation for $30 \mathrm{~min}$ at room temperature, $3 \mathrm{ml}$ of $3 \%$ ethylene glycol was added and the mixture was agitated for another $30 \mathrm{~min}$. At the same time, $100 \mathrm{mg}$ of BSA was dissolved in $20 \mathrm{ml}$ of $0.5 \mathrm{~m}$ sodium carbonate buffer (pH 9.6) and subsequently reacted with the former reaction solution overnight at $4{ }^{\circ} \mathrm{C}$. Then, $20 \mathrm{ml}$ of $\mathrm{NaBH}_{3} \mathrm{CN}$ $(500 \mathrm{mg})$ was added and incubation was continued overnight at $4{ }^{\circ} \mathrm{C}$. Finally, the reaction mixture was dialyzed against distilled water (6 changes over $24 \mathrm{~h}$ ) and applied to a column of sephadex G-100 equilibrated with $0.2 \mathrm{~m}$ ammonium bicarbonate. The column was eluted with $0.02 \mathrm{M}$ sodium phosphate buffer, $\mathrm{pH} 7.0$ (PBS). The elution was monitored at $280 \mathrm{~nm}$ for BSA, and determined by phenol-sulfuric acid reaction for MPS. Fractions positive for both protein and sugar, namely MPS-BSAp, were pooled, dialyzed exten- 
sively against distilled water and lyophilized. Quantitative protein (based on A280 and a BSA standard curve) and carbohydrate (determined by phenol-sulfuric acid reaction) analysis showed that the conjugate contained carbohydrate and BSA in approximate a $4: 1$ molar ratio.

Preparation of MPS-BSAr by Reductive Amination Method The reducing terminus of MPS has a reactive aldehyde through which MPS can be coupled by reductive amination to amino groups in BSA. Briefly, $500 \mathrm{mg}$ of MPS and $100 \mathrm{mg}$ of BSA were dissolved in $20 \mathrm{ml}$ of $0.2 \mathrm{M}$ potassium phosphate buffer, $\mathrm{pH} 8.0$. After addition of $500 \mathrm{mg}$ of $\mathrm{NaBH}_{3} \mathrm{CN}$, the reaction mixture was left at $37^{\circ} \mathrm{C}$ for $3 \mathrm{~d}$. The mixture was then applied to a column of sephadex G-100 equilibrated and washed with deionized water. The elution was monitored at $280 \mathrm{~nm}$ for BSA, and determined by phenol-sulfuric acid reaction for carbohydrate. Fractions positive for both protein and sugar, namely MPS-BSAr, were pooled and lyophilized. Quantitative protein (based on A280 and a BSA standard curve) and carbohydrate (determined by phenol-sulfuric acid reaction) analysis showed that the conjugate contained carbohydrate and BSA in approximate a $2: 1$ molar ratio. MPS-OVA conjugate was prepared using the same method.

Generation of Antibodies Antibodies against MPS were obtained following traditional methods. Briefly, female $\mathrm{Balb} / \mathrm{c}$ mice aged $6-8$ weeks were immunized with MPS-BSAp and MPS-BSAr conjugates respectively. A volume of $0.25 \mathrm{ml}$ of $800 \mu \mathrm{g} / \mathrm{ml}$ of conjugate solution mixed with $0.25 \mathrm{ml}$ of complete Freund's adjuvant was given subcutaneously on day 1 . On day $12,0.25 \mathrm{ml}$ conjugate solution mixed with $0.25 \mathrm{ml}$ of incomplete Freund's adjuvant was given subcutaneously. Then on days 20 and $28,0.25 \mathrm{ml}$ of conjugate solution was given intraperitoneally. One week later, mice sera were collected and detected by ELISA. The spleens from mice with high titer antisera were chosen to prepare hybridomas. The fusion and cloning procedures were performed according to an established protocol. ${ }^{18)}$

Indirect ELISA to Detect Antibodies against MPS The single steps of the assay procedure were performed according to general ELISA tests. ${ }^{19,20)}$ The special conditions are briefly as follows: microtiter plates were coated with $100 \mu \mathrm{l} /$ well conjugate solution or MPS solution at a concentration of $100 \mu \mathrm{g} / \mathrm{ml}$ in $0.05 \mathrm{M}$ sodium bicarbonate buffer, $\mathrm{pH}$ 8.5 , overnight at $4{ }^{\circ} \mathrm{C}$. After washing three times with a washing buffer (PBS containing $0.05 \%$ Tween-20), the plates were blocked by incubation with $1 \%$ gelatin in PBS, for $2 \mathrm{~h}$ at $37^{\circ} \mathrm{C}$. Next, the plates were washed three times with washing buffer.

To neutralize BSA antibody, mice sera were diluted 1/100 in PBS containing $1 \%$ BSA followed by incubation for $30 \mathrm{~min}$ at $37^{\circ} \mathrm{C}$, and then centrifuged for $5 \mathrm{~min}$ at $3000 \mathrm{rpm}$. The supernatants diluted in 2-fold were added to microtiter plates $(50 \mu \mathrm{l} /$ well $)$ in triplicate and incubated for $1 \mathrm{~h}$ at $37^{\circ} \mathrm{C}$. Sera from normal mice prepared in the same way were used as negative controls. The plates were incubated for another $1 \mathrm{~h}$ at $37^{\circ} \mathrm{C}$ with HRP conjugated goat anti-mouse IgG antibody diluted $1 / 10000$ in PBS containing $0.05 \%$ Tween-20 and $1 \%$ BSA $(50 \mu \mathrm{l} /$ well $)$. Each incubation step was terminated by washing the microtiter plates with washing buffer as previously described. Finally, $0.05 \%$ of the HRP substrate TMB solution was added $(100 \mu \mathrm{l} /$ well). The reaction was stopped with $0.5 \mathrm{M} \mathrm{H}_{2} \mathrm{SO}_{4}(100 \mu \mathrm{l} /$ well $)$ after a $15 \mathrm{~min}$ incubation at $37^{\circ} \mathrm{C}$. The absorbance values were recorded at $450 \mathrm{~nm}$ on an ELISA reader. The positive titer was defined as the dilution that gave an optical density of 2.1 times negative control.

Competitive ELISA MPS was added to the microtiter plates pre-coated with MPS-BSA conjugates in 10-fold PBS dilutions from $100 \mu \mathrm{g} /$ well $(50 \mu \mathrm{l} /$ well $)$, and the supernatants of mice sera diluted $1 / 100$ in PBS containing $1 \%$ BSA were added simultaneously. The microtiter plates were incubated for $1 \mathrm{~h}$. Sera from normal mice diluted 1/100 were used as negative controls. The plates were incubated for another $1 \mathrm{~h}$ at $37^{\circ} \mathrm{C}$ with HRP conjugated secondary antibodies diluted $1 / 10000$ in PBS containing $0.05 \%$ Tween-20 and $1 \%$ BSA ( $50 \mu \mathrm{l} /$ well). Each incubation step was terminated by washing the microtiter plates with washing buffer as previously described. Finally, $0.05 \%$ of the HRP substrate TMB solution was added $(100 \mu \mathrm{l} /$ well). The reaction was stopped with $0.5 \mathrm{M} \mathrm{H}_{2} \mathrm{SO}_{4}(100 \mu \mathrm{l} /$ well $)$ after $15 \mathrm{~min}$ incubation at $37^{\circ} \mathrm{C}$. The absorbance values were recorded at $450 \mathrm{~nm}$ on an ELISA reader. The competition between the antigen preparations in solution and the bound antigen was seen as a reduction in absorbance compared to that obtained in the absence of antigen in solution.

\section{RESULTS}

\section{Detection of Antibodies Induced by MPS-BSAp with}
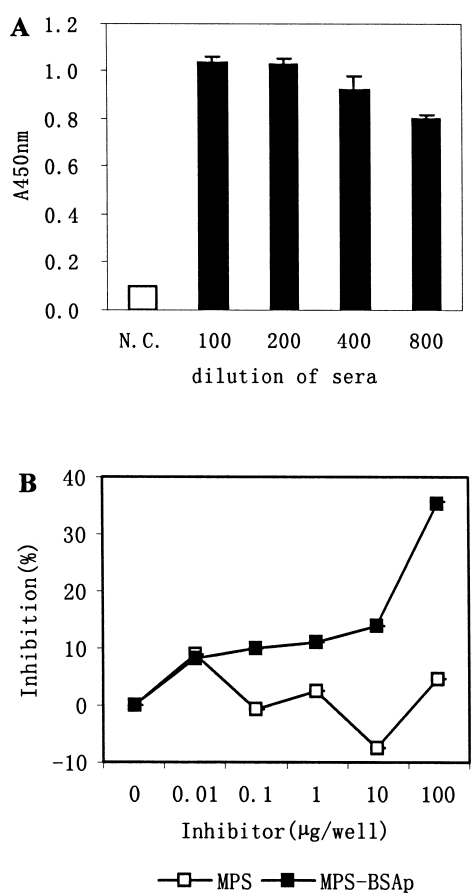

Fig. 1. ELISA of Sera Samples from Mice Immunized with MPS-BSAp

(A) Indirect ELISA using microplates coated with MPS-BSAp. A 50- $\mu$ l volume of normal mice sera diluted $1 / 100$ was used as negative control (N.C.). A $50-\mu 1$ volume of sera samples was 2-fold diluted starting from 1/100. (B) Competitive ELISA of MPS, MPS-BSAp using microplates coated with MPS-BSAp. A 50- $\mu$ 1 volume of MPS or MPS-BSAp conjugate solutions diluted in 10-fold starting from $100 \mu \mathrm{g} / \mathrm{well}$ was added to the plates. A $50-\mu 1$ volume of sera samples diluted $1 / 100$ was subsequently added to the plates. Absorbance values were converted into inhibition (\%) values using the equation inhibition $(\%)=[1-(\mathrm{A}-\mathrm{An}) /(\mathrm{Ao}-\mathrm{An})] \times 100$, where $\mathrm{A}$ represents values in the presence of inhibitor, while Ao is the absorbance obtained without inhibitor, and An is the absorbance of negative control. 
Indirect and Competitive ELISA Using microplates coated with MPS, detection of sera from mice immunized with MPS-BSAp was negative (data not shown). While using microplates coated with MPS-BSAp, the dilutions of sera were up to $1 / 800$ (Fig. 1A). Two factors might be responsible for the improved dilutions with MPS-BSAp as an immobilizing antigen. Firstly, the sensitivity was enhanced for higher immobilization efficiency because direct adsorption of low molecular weight polysaccharide on the surface of polyethylene microplates might be inefficient. Secondly, there were antibodies not against MPS but rather for periodate-modified MPS.

To confirm the specificity of antibodies produced by mice immunized with MPS-BSAp, the competitive ELISAs of MPS and MPS-BSAp conjugate were performed. As shown in Fig. 1B, at the concentration of $100 \mu \mathrm{g} /$ well, MPS in solution still could not compete with the bound antigen, while the inhibition of MPS-BSAp was above $30 \%$. There was a significant difference between the inhibition profiles of these two inhibitors, and only MPS-BSAp exerted competitive inhibition. The results demonstrated that there existed antibodies only specific for MPS-BSAp, but not for native MPS in sera in accordance with the results from indirect ELISA, which might be attributed to the changes in epitopes of MPS during the coupling procedures by periodate oxidation. Moreover, the 30\% inhibiton of MPS-BSAp even at the concentration of $100 \mu \mathrm{g} /$ well might be due to the low affinity of antibodies or high titer antibodies in sera.

A very common technique for improving the immobilization of small molecules is to prepare conjugates with carrier proteins. ${ }^{15,21-23)}$ With an MPS-carrier conjugate as a coating antigen, the background of ELISA could be lowered leading to an improvement in the sensitivity. MPS-BSA conjugates were going to be used as coating antigens in our experiments.

In order to reduce the background absorbance values, BSA was generally added to sera samples. ${ }^{20,24)}$ Here, MPS-BSA conjugates were used as immunogens and coating antigens at the same time, following which BSA was added to neutralize anti-BSA antibodies in sera samples to avoid nonspecific absorption that would induce false positive effects. A series of concentrations of BSA were added to sera samples. The influence on ELISA absorbance values is shown in Fig. 2. One percent BSA was sufficient for neutralizing BSA antibodies in sera. When detecting antibodies induced by MPS-BSAr in the same method, a similar conclusion was obtained (data not shown). Therefore in our experiments, 1.0\% BSA was added to sera to neutralize anti-BSA antibodies. This experiment suggested to us that a carbohydrate-protein conjugate can be used as immunogen and coating antigen at the same time by adding protein to react with anti-protein antibodies in sera to avoid non-specific reaction when detecting sera by ELISA. This is a convenient and time-saving means in which only a carbohydrate-conjugate need be prepared.

Detection of Antibodies Induced by MPS-BSAr with Indirect and Competitive ELISA Using microplates absorbed directly with MPS, the dilution of sera from mice immunized with MPS-BSAr was to $1 / 200$ (Fig. 3A). Meanwhile as shown in Fig. 3B, the titer was above $1 / 800$ when using microplates coated with MPS-BSAr. Compared to $1 / 200$ of MPS-coated microplates, the sensitivity was remarkably augmented. These experiments illustrated that

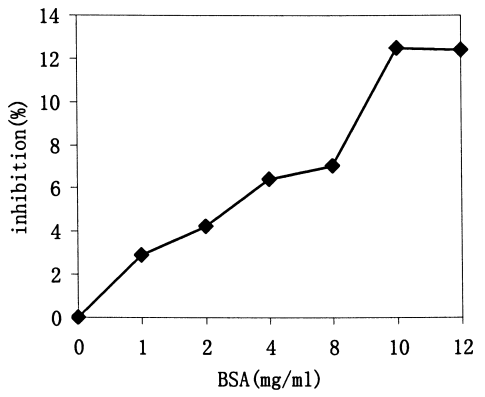

Fig. 2. Effects on ELISA Absorbance Values of Various Concentrations of BSA in Sera Samples

A $50-\mu 1$ volume of normal mice sera diluted $1 / 100$ was used as negative control (N.C.). Sera samples diluted $1 / 100$ with different concentrations of BSA were incubated for $30 \mathrm{~min}$ at $37^{\circ} \mathrm{C}$ and centrifuged for $5 \mathrm{~min}$ at $3000 \mathrm{rpm}$. A $50-\mu \mathrm{l}$ volume of the supernatants was added to plates coated with MPS-BSAp. Absorbance values were converted into inhibition (\%) values as described above.
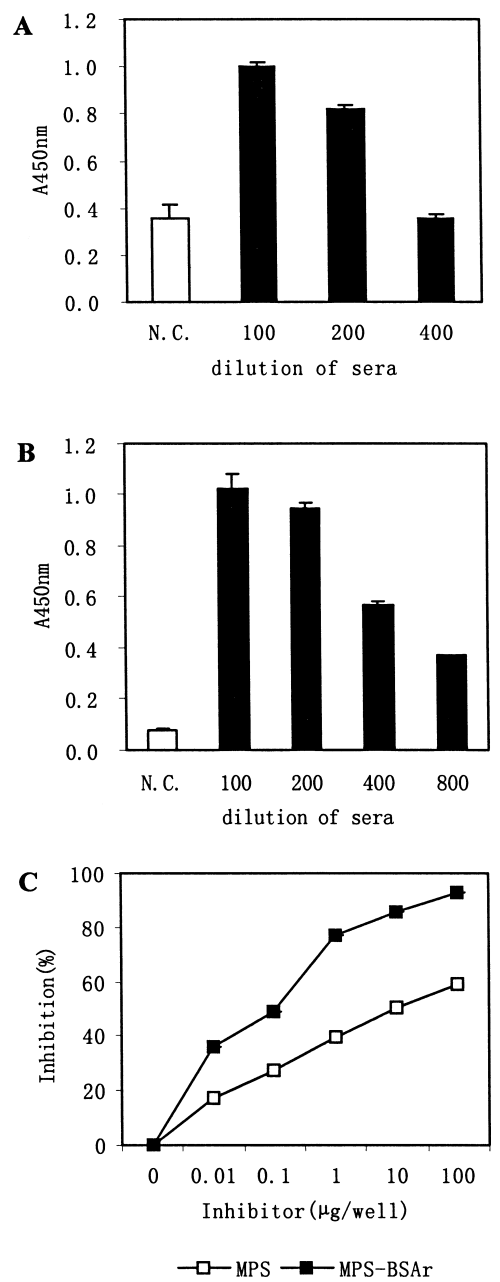

Fig. 3. ELISA of Sera Samples from Mice Immunized with MPS-BSAr

(A) Indirect ELISA of sera samples using microplates coated with MPS. (B) Indirect ELISA of sera samples using microplates coated with MPS-BSAr. A 50- $\mu 1$ volume of normal mice sera diluted $1 / 100$ was used as negative control (N.C.). A $50-\mu 1$ volume of sera samples was 2-fold diluted starting from 1/100. (C) Competitive ELISA of MPS, MPS-BSAr using microplates coated with MPS-BSAr. A 50- $\mu 1$ volume of MPS or MPS-BSAr conjugate solution diluted in 10-fold starting from $100 \mu \mathrm{g} /$ well was added to the plates. A $50-\mu 1$ volume of sera samples diluted $1 / 100$ was subsequently added to the plates. Absorbance values were converted into inhibition (\%) values as described above.

there were antibodies against MPS in sera from mice immunized with MPS-BSAr. In addition, MPS directly absorbed to polyethylene microplates was inefficient for ELISA. 


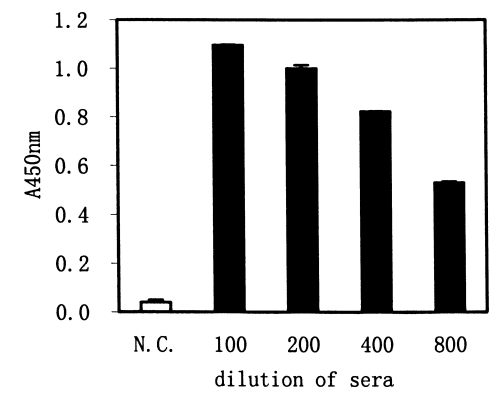

Fig. 4. Indirect ELISA of Sera Samples from Mice Immunized with MPS-BSAr Using Microplates Coated with MPS-OVA

A $50-\mu 1$ volume of normal mice sera diluted $1 / 100$ was used as negative control (N.C.). A $50-\mu$ l volume of sera samples was 2 -fold diluted starting from $1 / 100$.

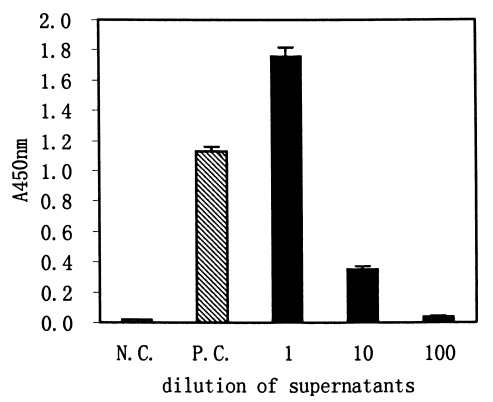

Fig. 5. Indirect ELISA of Hybridoma Cells Culture Supernatants Using Microplates Coated with MPS-OVA

A 50- $\mu 1$ volume of NS-1 myeloma cells culture supernatants was used as negative control (N.C.). A $50-\mu$ l volume of sera samples before fusion diluted $1 / 200$ was used as positive control (P.C.). A 50- $\mu$ l volume of hybridoma cells culture supernatants diluted 10 -fold in PBS containing $2 \%$ BSA was added to the plates.

MPS and MPS-BSAr conjugates were used as inhibitors in competitive ELISAs to further confirm the existence of antibodies specific for MPS (Fig. 3C). Both MPS and MPS-BSAr competitively inhibited the binding of antibodies to MPS-BSAr conjugate coated on the plates. At the concentration of $100 \mu \mathrm{g} /$ well, the inhibition of MPS was about $60 \%$, while the inhibition of MPS-BSAr was above $90 \%$. Though MPS-BSAr exerted higher inhibitions (\%) at the same concentrations, these two inhibitors had markedly similar inhibition profiles, from which the existence of antibodies for MPS could be deduced. Meantime, the higher inhibition of MPS-BSAr made the existence of antibodies for MPS-BSAr possible.

Indirect ELISA to Detect Antibodies with Plates Precoated with MPS-OVA In order to further confirm the results and to determine whether there existed antibodies against MPS-BSAr conjugate, MPS-OVA conjugate was used as the coating antigen in indirect ELISA to detect sera sample from mice immunized with MPS-BSAr. MPS-OVA conjugate was prepared also by reductive amination, but was reacted for $4 \mathrm{~d}$. Calculated as described above, the molar ratio of MPS to OVA in MPS-OVA conjugate was $7: 1$. Figure 4 shows that the ratio of absorbance values of sera samples diluted $1 / 800$ to normal mice sera was far higher than 2.1. This result ensured that the antibodies were specific for MPS, not for MPS-BSAr, and also indicated that a higher sensitivity would get with a higher molar ratio of MPS to protein conjugate as coating antigen. The advantage will be useful in hybridoma technology for the screening of mAbs.
Detection of Hybridoma Cell Culture Supernatants An MPS-BSAr-immunized mouse was chosen to prepare hybridomas against MPS. NS-1 myeloma cells were mixed with spleen cells from immunized mice at a ratio of 1 to 5 . The fusion and cloning procedures were performed as described previously. ${ }^{18)}$ As shown in Fig. 5, supernatants diluted 10-fold were still positive. Supernatants were also used in competitive ELISA of MPS, and indirect ELISA using microplates coated with BSA or OVA. It has been observed that free MPS competed with MPS coated on plates, and there were no cross-reactivities between supernatants and BSA or OVA (data not shown). The results proved that there were mAbs specific for MPS in supernatants, and that a cloning cell line secreting mAbs against MPS was established.

\section{DISCUSSION}

Initially, we intended to prepare mAb aganist MPS for biological and clinical applications, but low molecular weight polysaccharide MPS is a hapten that could not directly induce antibodies generation in animals, so Balb/c mice were immunized with two artificial immunogens, MPS-BSAp and MPS-BSAr. Sera samples from Balb/c mice were evaluated by ELISA. The results revealed that mice immunized with MPS-BSAr generated high titer antibodies against MPS only. Of note, mice immunized with MPS-BSAp generated antibodies against MPS-BSAp conjugate, not specific for MPS.

The reason why mice immunized with MPS-BSAp generated antibodies against MPS-BSAp conjugate rather than MPS per se might be explained by the fact that vicinal hydroxyl groups on the sugar unit of MPS were converted non-preferentially into aldehyde groups which were coupled to amino groups of BSA at random. During this process, BSA or polysaccharide might induce conformational changes, particularly responsible for the new epitopes formation in conjugate, which resulted in some differences in the epitopes of MPS-BSAp conjugate and MPS. Paloletti et al. suggested that the process of coupling capsular polysaccharide to protein by periodate oxidation might alter the conformation of polysaccharide. ${ }^{25)}$ Our experiments revealed this possibility was likely. Moreover, others had reported that animals immunized with heparin-protein conjugates produced antibodies for modified heparin, not for native heparin. ${ }^{11,16)}$

Therefore, in some cases, the efficiency of the coupling reaction has been increased by the introduction of functional groups at specific or random sites on carbohydrate. Such modifications of the carbohydrate, however, may alter the structure of the carbohydrate sufficiently to affect epitope expression, in which case the carbohydrate-protein conjugate may not elicit antibodies reactive with the native antigen. Therefore, an important prerequisite for the use of conjugates is that the antigen-antibody interaction remains sterically uninfluenced by the covalent attachment of the antigen to the carrier molecule. Thus, when carbohydrates are coupled to proteins to prepare conjugates used as immunogens, coupling method induced no alteration in determinants is the key requirement for generating specific antibodies against carbohydrates. 
In contrast, MPS-BSAr was preferentially coupled through a hemi-acetal group in reductive terminus of MPS and amino groups of BSA. MPS with only reductive terminus coupling to BSA was free from BSA, which influenced the epitopes of MPS to the least extent so there was no change in the specificity of $\mathrm{mAb}$. The results obtained in our experiments support the possibility that the method of reductive amination may be more suitable for coupling low molecular weight polysaccharides such as MPS to carrier proteins as preference to preparing specific $\mathrm{mAb}$.

A high serum titer mouse immunized with MPS-BSAr was chosen to prepare mAb specific for MPS. A hybridoma cell line which secreted $\mathrm{mAb}$ recognizing MPS selectively and sensitively, with no cross reactivities with mucopolysaccharides such as heparin, heparan sulfate etc. (data not shown), was established. The potential interests of this monoclonal MPS antibody may be widely used in biological and clinical studies in the near future.

Acknowledgements This work was funded by Chinese High-tech Project "863" (2001AA624140 and 2001AA628110). We thank Dr. Fuchuan Li and Dr. Kun Chen for their helpful suggestions during the experiments.

\section{REFERENCES}

1) Glennie M. J., Johnson P. W. M., Immunol. Today, 21, 403-410 (2000).

2) Straus A. H., Travassos L. R., Takahashi H. K., Analytical Biochem., 201, 1-8 (1992).

3) Huhle G., Harenberg J., Malsch R., Heene D., Semin. Thromb. Hemostas., 23, 17-21 (1997).

4) Pawlowski A., llenius G. K., Svenson S. B., Vaccine, 17, 1474-1483 (1999).

5) Caterson B., Christner J. E., Baker J. R., J. Biol. Chem., 258, 8848 8854 (1983).
6) Yamagata M., Kimata K., Oike K., Tani K., Maeda N., Yoshida K., Shimomura Y., Yoneda M., Suzuki S., J. Biol. Chem., 262, 41464152 (1986).

7) Van den Born J., Van den Heuvel L. P. W. J., Bakker M. A. H., Veerkamp J. H., Assmann K. J. M., Berden J. H. M., Lab. Invest., 65, 287-297 (1991).

8) Eckert T. F., Kozel T. R., Infection and Immunity, 55, 1895-1899 (1987).

9) Casadevall A., Mukherjee J., Devi S. J. N., Schneerson R., Robbins J. B., Scharff M. D., J. Infectious Diseases, 165, 1086-1093 (1992).

10) Ikeda R., Nishimura S., Nishikawa A., Shinoda T., Clin. Diagn. Lab. Immunol., 3, 89-92 (1996).

11) Gitel S. N., Medina V. M., Wessler S., Blood, 65, 902-911 (1985).

12) Bernard D., Nicolas C., Maurizis J. C., Betail G., J. Immunol. Methods, 61, 351-357 (1983).

13) Böcher M., Böldicke T., Kieß M., Bilitewski U., J. Immunol. Methods, 208, 191-202 (1997).

14) Wessels M. R., Paoletti L. C., Kasper D. L., DiFabio J. L., Michon F. Holme K., Jennings H. J., J. Clin. Invest., 86, 1428-1433 (1990).

15) Jung D. W., Sung C. K., Arch. Pharm. Res., 21, 135-139 (1998).

16) Pejler G., Lindahl U., Larm O., Scholander E., Sandgren E., J. Biol. Chem., 263, 5197-5201 (1988).

17) Huhle G., Harenberg J., Malsch R., Heene D. L., Semin. Thromb. Hemost., 20, 193-204 (1994).

18) De St Groth F. S., Scheidagger D., J. Immunol. Methods, 35, $1-21$ (1980).

19) Schrijver R. S., Kramps J. A., Rev. Sci. Tech. Off. Int. Epiz., 17, 550 (1998).

20) Jauho E. S., Boas U., Wiuff C., Wredstrom K., Pedersen B., Andresen L. O., Heegaard P. M. H., Jakobsen M. H., J. Immunol. Methods, 242, 133-143 (2000).

21) Briand J. P., Muller S., Van Regenmortel M. H. V., J. Immunol. Methods, 78, 59-69 (1985)

22) Edwards R. J., Singleton A. M., Boobis A. R., Davies D. S., J. Immunol. Methods, 117, 215-220 (1989).

23) Yu Z., Carter J. M., Sigal L. H., Stein S., J. Immunol. Methods, 198, 25-33 (1996).

24) Nielsen B., Baggesen D., Bager F., Haugegaard J., Lind P., Vet. Microbiol., 47, 205 (1995).

25) Paoletti L. C., Wessels M. R., Michon F., Difabioe J., Jennings H. J., Kasper D. L., Infection and Immunity, 10, 4009-4014 (1992) 